\title{
TENSOR PRODUCTS AND GROTHENDIECK TYPE INEQUALITIES OF OPERATORS IN $L_{p}$-SPACES
}

\author{
BERND CARL AND ANDREAS DEFANT
}

\begin{abstract}
Several results in the theory of $(p, q)$-summing operators are improved by a unified but elementary tensor product concept.
\end{abstract}

\section{INTRODUCTION}

Since the pioneering work of Grothendieck in Linear Functional Analysis there is an extensive literature dealing with operators in $L_{p}$-spaces. Still the most important result in this direction is Grothendieck's integral characterization [9] of operators from $l_{1}$ into $l_{2}$ : There is a universal constant $K_{G}>0$ such that for every operator $S: l_{1} \rightarrow l_{2}$ there is a probability measure $\nu$ on the unit ball $B_{l_{\infty}}$ of $l_{\infty}$ (endowed with its weak ${ }^{*}$ topology) for which

$$
\|S x\| \leq K_{G}\|S\| \int|\langle x, a\rangle| d \nu(a)
$$

holds for all $x \in l_{1}$. For information on estimates of the constant $K_{G}$ we refer to [20]. This result which is now called Grothendiek's Theorem-Grothendieck himself called it "the fundamental theorem of the metric theory of tensor products"-motivated the following statement of this paper: there is an absolute constant $k>0$ such that for every operator $S: l_{1} \rightarrow l_{v}(1 \leq v \leq \infty)$ and every probability measure $\mu$ on $B_{l_{v^{\prime}}}\left(v^{\prime}\right.$ the conjugate index $\left.v /(v-1)\right)$ there is a probability measure $\nu$ on $B_{l_{\infty}}$ with

$$
\left(\int|\langle S x, a\rangle|^{s} d \mu(a)\right)^{1 / s} \leq k\|S\| \int|\langle x, a\rangle| d \nu(a) ;
$$

for all $x \in l_{1}$, where $2 \leq s \leq \infty$ and $\frac{1}{s}=\left|\frac{1}{2}-\frac{1}{v}\right|$. Operators satisfying such integral inequalities were defined and intensively studied by Maurey [16] who proved several deep equivalent characterizations. One of them combined with our result states that every operator $S: l_{1} \rightarrow l_{v} \quad(1 \leq v \leq \infty)$ maps an unconditional summable sequence $\left(x_{i}\right)$ in $l_{1}$ into a sequence $\left(S x_{i}\right)$ which is the product of an absolutely $s^{\prime}$-summable scalar sequence $\left(\alpha_{i}\right)$ and a weakly $s$ summable sequence $\left(y_{i}\right)$ in $l_{v}$, here again $2 \leq s \leq \infty$ and $\frac{1}{s}=\left|\frac{1}{2}-\frac{1}{v}\right|$. Within the theory of absolutely summing operators which was initiated by Pietsch [22], our result has the following formulation: If $2 \leq s \leq \infty$ and $\frac{1}{s}=\left|\frac{1}{2}-\frac{1}{v}\right|$,

Received by the editors March 6, 1989 and, in revised form, January 10, 1990.

1980 Mathematics Subject Classification (1985 Revision). Primary 46B20, 47B10, 46M05. 
then the composition of an arbitrary operator $S: l_{1} \rightarrow l_{v}$ with an absolutely $s$-summing operator $T: l_{v} \rightarrow E$ is already absolutely summing.

The proof is based on two powerful concepts, which at a first glance have nothing in common with each other, and also Kwapien's important extension [12] of Grothendieck's Theorem, namely: If $1 \leq v \leq \infty$ and $\frac{1}{r}=1-\left|\frac{1}{2}-\frac{1}{v}\right|$, then every operator $S: l_{1} \rightarrow l_{v}$ is absolutely $(r, 1)$-summing, i.e., it transforms every unconditional summable sequence of $l_{1}$ into an absolutely $r$-summable sequence of $l_{v}$. Our first step is to use Kwapien's result and the concept of Weyl numbers (treated in König [11] and Pietsch [23]) to obtain some weaker estimates concerning our statement. The next step is to improve these estimates by a general procedure based on a certain kind of tensor multiplicativity of some operator norms with respect to tensor products of $L_{p}$-spaces.

Along similar lines we study identity operators $I$ from $l_{u}$ into $l_{v} \quad(1 \leq$ $u \leq v \leq \infty)$. In particular, we prove for $I: l_{u} \hookrightarrow l_{2}$ the following integral characterization: there is a constant $k(u)>0$ (depending just on $u$ ) such that for every probability measure $\mu$ on $B_{l_{2}}$ there is a probability measure $\nu$ on $B_{l_{u^{\prime}}}$ for which

$$
\left(\int|\langle x, a\rangle|^{u^{\prime}} d \mu(a)\right)^{1 / u^{\prime}} \leq k(u) \int|\langle x, a\rangle| d \nu(a)
$$

holds for all $x \in l_{u}$. This is a generalization of a well-known characterization of absolutely $(r, 1)$-summing identity operators $I: l_{u} \hookrightarrow l_{2}$ due to Bennett [2] and (independently) the first author [4], which itself extends old results of Hardy and Littlewood [10] on continuous bilinear forms on $l_{p} \times l_{q} \quad(1 \leq p, q \leq \infty)$.

Moreover, we give new integral descriptions of Schatten-von Neumann operators of type $l_{r}$.

The paper is divided into two main parts; in the first part we recall the basic definitions and results of Maurey's theory of $(s, p)$-mixing operators and develop our basic tools; in the second part we prove the above-mentioned integral characterizations of operators acting between $L_{p}$-spaces.

\section{1. $(s, p)$-MIXING OPERATORS, WEYL NUMBERS AND TENSOR MULTIPLICATIVITY}

Let us start with some preliminaries. We shall use standard notations and notions from Banach space theory, as presented in [15]. For the general theory of Banach operator ideals which was founded by Pietsch, we refer the reader to the monograph [22].

If $E$ is a Banach space (over the scalar field $\mathbb{K}=\mathbb{R}$ or $\mathbb{C}$ ), then $B_{E}$ is its (closed) unit ball and $E^{\prime}$ its dual. By $W\left(B_{E^{\prime}}\right)$ we denote the set of all (regular Borel) probability measures on the weak ${ }^{*}$-compact space $B_{E^{\prime}}$. A family $\left(x_{i}\right)$ in $E$ is called absolutely $p$-summable $(0<p \leq \infty)$ if

$$
l_{p}\left(x_{i}\right):=l_{p}\left(x_{i} ; E\right):=\left(\sum_{i}\left\|x_{i}\right\|^{p}\right)^{1 / p}<\infty,
$$

and weakly $p$-summable if

$$
w_{p}\left(x_{i}\right):=w_{p}\left(x_{i} ; E\right):=\sup \left\{\left(\sum_{i}\left|\left\langle x_{i}, a\right\rangle\right|^{p}\right)^{1 / p} \mid a \in B_{E^{\prime}}\right\}<\infty
$$


(with the obvious modifications for $p=\infty)$. By an $L_{p}$-space $(1 \leq p \leq \infty)$ we mean a Banach space $L_{p}(\Omega, \Sigma, \mu)$ for some measure space $(\Omega, \Sigma, \mu)$. The class of all $L_{p}$-spaces includes $l_{p}$ and all spaces $l_{p}^{n}\left(:=\mathbb{K}^{n}\right.$ equipped with the norm $\left.l_{p}(\cdot)\right)$.

As usual $\mathscr{L}(E, F)$ denotes the Banach space of all (bounded and linear) operators $S$ from $E$ to $F$ endowed with the operator norm

$$
\|S\|:=\sup \left\{\|S x\| \mid x \in B_{E}\right\} \text {. }
$$

An operator $T \in \mathscr{L}(E, F)$ is called absolutely $(r, p)$-summing $(0<p \leq$ $r \leq \infty)$ if there is a constant $\rho \geq 0$ such that $l_{r}\left(T x_{i}\right) \leq \rho w_{p}\left(x_{i}\right)$, for all finite sets of elements $x_{1}, \ldots, x_{n} \in E$. In this case the infimum over all possible $\rho \geq 0$ is denoted by $\pi_{r, p}(T)$. Then $\left[\mathscr{P}_{r, p}, \pi_{r, p}\right]$ is a $[\min \{p, 1\}]$-normed Banach ideal of operators.

By a result of Kwapien

$$
\left[\mathscr{P}_{r_{1}, p_{1}}, \pi_{r_{1}, p_{1}}\right] \subseteq\left[\mathscr{P}_{r_{2}, p_{2}}, \pi_{r_{2}, p_{2}}\right]
$$

if $r_{1} \leq r_{2}, p_{1} \leq p_{2}$ and $1 / r_{1}-1 / p_{1}=1 / r_{2}-1 / p_{2}$. If $r=p$ one gets the ideal $\left[\mathscr{P}_{p}, \pi_{p}\right]$ of all absolutely $p$-summing operators. The Grothendieck-Pietsch domination theorem states that $S \in \mathscr{P}_{p}(E, F)(0<p \leq \infty)$ if and only if there is a $\rho \geq 0$ and a $\nu \in W\left(B_{E^{\prime}}\right)$ for which

$$
\|S x\| \leq \rho\left(\int|\langle x, a\rangle|^{p} d \nu(a)\right)^{1 / p}
$$

holds for each $x \in E$. In this case again $\pi_{p}(T)=\min \rho$. Moreover, we mention Pietsch's composition formula for absolutely summing operators:

$$
\left[\mathscr{P}_{s} \cdot \mathscr{P}_{r}, \pi_{s} \cdot \pi_{r}\right] \subseteq\left[\mathscr{P}_{p}, \pi_{p}\right], \quad \frac{1}{s}+\frac{1}{r}=\frac{1}{p} \leq 1 .
$$

Let us finally recall the definition of a $p$-nuclear operator. An operator $T \in$ $\mathscr{L}(E, F)$ is said to be $p$-nuclear $(1 \leq p \leq \infty)$ if it admits a representation

$$
T=\sum_{i=1}^{\infty} a_{i} \otimes y_{i}
$$

with $l_{p}\left(a_{i} ; E^{\prime}\right) w_{p^{\prime}}\left(y_{i} ; F\right)<\infty$. Put $N_{p}(T):=\inf l_{p}\left(a_{i}\right) w_{p^{\prime}}\left(y_{i}\right)$. Then the class $\mathscr{N}_{p}$ of all $p$-nuclear operators together with $N_{p}$ defines a Banach ideal of operators and

$$
\left[\mathscr{N}_{p}, N_{p}\right] \subseteq\left[\mathscr{P}_{p}, \pi_{p}\right], \quad 1 \leq p \leq \infty .
$$

1.1. A brief résumé of Maurey's theory of $(s, p)$-mixing operators. Operators of the following type for the first time were investigated in Maurey's fundamental thesis [16]:

An operator $S \in \mathscr{L}(E, F)$ is called $(s, p)$-mixing $(0<p \leq s<\infty)$ if there is a constant $\rho \geq 0$ such that for every probability measure $\mu \in W\left(B_{F^{\prime}}\right)$ there is a probability measure $\nu \in W\left(B_{E^{\prime}}\right)$ with

$$
\left.\left(\int|\langle S x, a\rangle|^{s} d \mu(a)\right)^{1 / s} \leq \rho\left(\int|\langle x, a\rangle|^{p} d \nu(a)\right)^{1 / p}\right),
$$

for all $x \in E$.

With $\mu_{s, p}(S):=\inf \rho=\min \rho$ the class $\mathscr{M}_{s, p}$ of all $(s, p)$-mixing operators forms a $[\min \{p, 1\}]$-normed Banach ideal. Obviously $\left[\mathscr{M}_{p, p}, \mu_{p, p}\right]=$ 
$[\mathscr{L},\|\cdot\|]$ and because of the domination theorem it makes sense to define $\left[\mathscr{M}_{\infty, p}, \mu_{\infty, p}\right]:=\left[\mathscr{P}_{p}, \pi_{p}\right]$ for $0<p \leq \infty$.

We now recall some basic results about $(s, p)$-mixing operators most of which can be found at least implicitly in Maurey's thesis [16] (see also [17]). In a condensed form the theory of these operators appeared in Pietsch [22] and Puhl [24]. Most of the following results will be used throughout this paper.

The phrase " $(s, p)$-mixing" refers to the following deep characterization by Maurey [16]: an operator $S \in \mathscr{L}(E, F)$ is $(s, p)$-mixing if and only if it maps every weakly $p$-summable scalar sequence $\left(x_{i}\right)$ in $E$ into a sequence which can be written as a product $\left(\alpha_{i} y_{i}\right)$ of an absolutely $r$-summable scalar sequence $\left(\alpha_{i}\right)$ and a weakly $s$-summable sequence $\left(y_{i}\right)$ in $F$, where $\frac{1}{s}+\frac{1}{r}=\frac{1}{p}$.

The following very useful composition formula is an immediate consequence of the definition: for $0<p \leq s \leq t \leq \infty$

$$
\left[\mathscr{M}_{t, s} \cdot \mathscr{M}_{s, p}, \mu_{t, s} \cdot \mu_{s, p}\right] \subseteq\left[\mathscr{M}_{t, p}, \mu_{t, p}\right] .
$$

Furthermore, we need a "local" version of the definition of an $(s, p)$-mixing operator: By the Grothendieck-Pietsch domination theorem it is easy to see that an operator $S \in \mathscr{L}(E, F)$ is $(s, p)$-mixing $(0<p \leq s<\infty)$ if and only if for every $\mu \in W\left(B_{F^{\prime}}\right)$ the mapping

$$
\begin{aligned}
& E \rightarrow F \rightarrow L_{s}\left(B_{F^{\prime}}, \mu\right) \\
& x \rightsquigarrow(a \rightsquigarrow\langle S x, a\rangle)
\end{aligned}
$$

is absolutely $p$-summing, i.e, there is $\rho \geq 0$ such that for all $x_{1}, \ldots, x_{n} \in E$

$$
\left(\sum_{i=1}^{n}\left(\int\left|\left\langle S x_{i}, a\right\rangle\right|^{s} d \mu(a)\right)^{p / s}\right)^{1 / p} \leq \rho w_{p}\left(x_{i}\right) .
$$

Since the discrete probabilities are (weak*-)dense in $W\left(B_{F^{\prime}}\right)$ this implies that $S \in \mathscr{L}(E, F)$ is $(s, p)$-mixing iff there is a constant $\rho \geq 0$ such that for all finite families of elements $x_{1}, \ldots, x_{n} \in E$ and functionals $b_{1}, \ldots, b_{m} \in F^{\prime}$

$$
\left(\sum_{i=1}^{n}\left(\sum_{k=1}^{m}\left|\left\langle S x_{i}, b_{k}\right\rangle\right|^{s}\right)^{p / s}\right)^{1 / p} \leq \rho w_{p}\left(x_{i}\right) l_{s}\left(b_{k}\right) .
$$

Again the infimum over all possible $\rho$ equals $\mu_{s, p}(S)$ (compare with [22, 20.1.4]).

Now we recall some basic examples. An immediate consequence of the domination theorem is the following quotient formula

$$
\left[\mathscr{M}_{s, p}, \mu_{s, p}\right]=\left[\mathscr{P}_{s}^{-1} \cdot \mathscr{P}_{p}, \pi_{s}^{-1} \cdot \pi_{p}\right], \quad 1 \leq p \leq s \leq \infty .
$$

In other words, an operator $S \in \mathscr{L}(E, F)$ is $(s, p)$-mixing iff its composition with an arbitrary operator $T \in \mathscr{P}_{s}(F, Y)$ is absolutely $p$-summing, and in this case

$$
\mu_{s, p}(S)=\sup \left\{\pi_{p}(T S) \mid \pi_{s}(T) \leq 1\right\}
$$

(see [22, 20.3.1]). Hence Pietsch's composition formula of absolutely summing operator implies

$$
\left[\mathscr{P}_{r}, \pi_{r}\right] \subseteq\left[\mathscr{M}_{s, p}, \mu_{s, p}\right], \quad \frac{1}{s}+\frac{1}{r}=\frac{1}{p} \leq 1 .
$$


Moreover, the preceding quotient formula can be dualized as follows (cf. [22, 20.3.2])

$$
\left[\mathscr{M}_{s, p}, \mu_{s, p}\right]=\left[\mathscr{N}_{s^{\prime}} \cdot \mathscr{N}_{p^{\prime}}^{-1}, N_{s^{\prime}} \cdot N_{p^{\prime}}^{-1}\right], \quad 1 \leq p \leq s \leq \infty,
$$

i.e., an operator $S \in \mathscr{L}(E, F)$ is $(s, p)$-mixing iff $S T \in \mathscr{N}_{s^{\prime}}(X, F)$ for every operator $T \in \mathscr{N}_{p^{\prime}}(X, E)$, and in this case

$$
\mu_{s, p}(S)=\sup \left\{N_{s^{\prime}}(S T) \mid N_{p^{\prime}}(T) \leq 1\right\} .
$$

The next result again due to Maurey [16] shows that the notions of $(s, p)$-mixing and absolutely $(r, p)$-summing operators are closely related. One has

$$
\left[\mathscr{M}_{s, p}, \mu_{s, p}\right] \subseteq\left[\mathscr{P}_{r, p}, \pi_{r, p}\right], \quad \frac{1}{s}+\frac{1}{r}=\frac{1}{p} ;
$$

(for an easy direct proof see [24]) and for $0<p<s<2$ this inclusion is strict. However, for $1 \leq p \leq s_{0}<s \leq \infty$ and $\frac{1}{s}+\frac{1}{r}=\frac{1}{p}$

$$
\left[\mathscr{P}_{r, p}, \pi_{r, p}\right] \subseteq\left[\mathscr{M}_{s_{0}, p}, \mu_{s_{0}, p}\right]
$$

(cf. [22, 20.1.11 and 20.1.12]).

Finally, we mention that the identity operator on every $L_{q}$-space $(1 \leq q \leq 2)$ is $(2, p)$-mixing $(0<p \leq 2)$. This result is due to Kwapien [13] and was extended by Maurey [16]:

$$
\mathrm{id}_{E} \in \mathscr{M}_{2, p} \text { for all } 0<p \leq 2 \text {, if } E \text { has cotype } 2
$$

(compare also with $[22,21.4 .9$ and 20.1.15]). A Banach space $E$ has cotype $q(2 \leq q<\infty)$ if there is a constant $c \geq 0$ such that for finitely many $x_{1}, \ldots, x_{n} \in E$

$$
\left(\sum_{i=1}^{n}\left\|x_{i}\right\|^{q}\right)^{1 / q} \leq c\left(\int_{0}^{1}\left\|\sum_{i=1}^{n} r_{i}(t) x_{i}\right\|^{2} d t\right)^{1 / 2},
$$

where $r_{i}$ is the $i$ th Rademacher function. Khinchine's inequality shows that every $L_{q}$-space $(1 \leq q<\infty)$ has cotype $\max \{q, 2\}$. For further information on this notion we refer to [20].

For a Banach space $E$ with $\operatorname{id}_{E} \in \mathscr{M}_{s, p}$ we put $\mu_{s, p}(E):=\mu_{s, p}\left(\mathrm{id}_{E}\right)$. The following estimates are known (cf. [22, 22.3.6])

$$
\mu_{2, p}\left(l_{q}\right) \leq \begin{cases}c_{2 q^{\prime}} c_{2 p}^{-1} & \text { for } 1<q \leq 2,0<p<2, \\ c_{2 p^{\prime}} c_{2 q}^{-1} & \text { for } 1 \leq q \leq 2,1<p<2,\end{cases}
$$

where

$$
\begin{gathered}
c_{2 p}=2\left[\frac{\Gamma\left(\frac{1+p}{2}\right)}{\Gamma\left(\frac{1}{2}\right)}\right]^{1 / p}, \quad c_{22}=\sqrt{2}, \quad c_{21}=\frac{2}{\sqrt{\pi}}(\mathbb{K}=\mathbb{R}), \\
C_{2 p}=2\left[\Gamma\left(\frac{2+p}{2}\right)\right]^{1 / p}, \quad c_{22}=2, \quad c_{21}=\sqrt{\pi}(\mathbb{K}=\mathbb{C}) .
\end{gathered}
$$

1.2. Mixing operators and Weyl numbers. The following lemma combines the theory of $(s, p)$-mixing operators with the concept of Weyl numbers. We just briefly recall some basic notions (for the general theory of $s$-numbers see the monographs $[11,23])$. 
The $n$th approximation number $a_{n}(S)$ of $S \in \mathscr{L}(E, F)$ is defined by

$$
a_{n}(S):=\inf \{\|S-T\| \mid T \in \mathscr{L}(E, F) \text { with rank } T<n\}
$$

and the $n$th Weyl number $x_{n}(S)$ is defined by

$$
x_{n}(S):=\sup \left\{a_{n}(S X) \mid E \in \mathscr{L}\left(l_{2}, E\right) \text { with }\|X\| \leq 1\right\} .
$$

Clearly, the sequences $\left(a_{n}(S)\right)$ and $\left(x_{n}(S)\right)$ are nonincreasing and $\|S\|=$ $a_{1}(S)=x_{1}(S)$. Moreover, they are multiplicative, i.e., $s_{n+m-1}(T S) \leq$ $s_{n}(T) s_{m}(S)$, for $s \in\{a, x\}, S \in \mathscr{L}(E, G), T \in \mathscr{L}(G, F)$, and $n, m \in \mathbb{N}$. by

Given $0<r<\infty$ and $0<q \leq \infty$ the Lorenz sequence space $l_{r, q}$ is defined

$$
l_{r, q}:=\left\{\xi \in l_{\infty} \mid l_{r, q}(\xi):=\left(\sum_{k=1}^{\infty} k^{q / r-1} \xi_{k}^{* q}\right)^{1 / q}<\infty\right\},
$$

where $\left(\xi_{n}^{*}\right)$ denotes the decreasing rearrangement of $\left(\left|\xi_{n}\right|\right)$. For $q=\infty$ the requirement is supposed to mean

$$
l_{r, \infty}(\xi):=\sup _{k \in \mathbb{N}} k^{1 / r} \xi_{k}^{*}<\infty .
$$

Clearly, $l_{r}:=l_{r, r}$. The spaces $l_{r, q}$ are ordered lexicographically. For $s \in$ $\{a, x\}$ and $0<r<\infty, 0<q \leq \infty$ let

$$
\mathscr{L}_{r, q}^{s}:=\left\{S \in \mathscr{L} \mid L_{r, q}^{s}(S):=l_{r, q}\left(\left(s_{n}(S)\right)\right)<\infty\right\}
$$

be the quasi Banach ideal of all operators with approximation numbers resp. Weyl numbers belonging to $l_{r, q}$.

We now establish a close relationship between the Banach ideal $\mathscr{M}_{s, 2}$ and certain Weyl number ideals. This result extends (and is also based on) the inclusions

$$
\mathscr{L}_{r, 1}^{x} \subseteq \mathscr{P}_{r, 2} \subseteq \mathscr{L}_{r, \infty}^{x}, \quad 2 \leq r<\infty,
$$

which are crucial for the theory of eigenvalue distribution of compact operators and go back to König, Lewis, Pisier and Pietsch (see e.g. [11, 2.a.11 or 23, 2.7.4]). Moreover,

$$
L_{r, \infty}^{x} \leq \pi_{r, 2} \text { and } \pi_{2} \leq 12 L_{2,1}^{x} .
$$

\subsubsection{Proposition. Let $\frac{1}{s}+\frac{1}{r}=\frac{1}{2}$.}

(1) $\mathscr{L}_{r, 1}^{x} \subseteq \mathscr{M}_{s, 2}$ and $\mu_{s, 2} \leq 24 L_{r, 1}^{x}$.

(2) For every finite rank operator $S, \mu_{s, 2}(S) \leq 24(1+\log (\operatorname{rank} S)) L_{r, \infty}^{x}(S)$.

Proof. In order to prove (1) by (1.1.3) it suffices to check $\mathscr{P}_{s} \cdot \mathscr{L}_{r, 1}^{x} \subseteq \mathscr{P}_{2}$.

Let $S \in \mathscr{L}_{r, 1}^{x}(E, F)$ and $T \in \mathscr{P}_{s}(F, Y) \subseteq \mathscr{P}_{s, 2}(F, Y) \subseteq \mathscr{L}_{s, \infty}^{x}(F, Y)$. By the monotonicity and multiplicativity of the Weyl numbers $T S \in \mathscr{L}_{2,1}^{x}$, since

$$
\begin{aligned}
\sum_{k=1}^{\infty} k^{-1 / 2} x_{k}(T S) & \leq 2 \sum_{k}(2 k-1)^{-1 / 2} x_{k}(T) x_{k}(S) \\
& \leq 2 L_{s, \infty}^{x}(T) L_{r, 1}^{x}(S) \\
& \leq 2 \pi_{s}(T) L_{r, 1}^{x}(S) .
\end{aligned}
$$

Hence $T S \in \mathscr{P}_{2}$ and

$$
\pi_{2}(T S) \leq 12 L_{2,1}^{x}(T S) \leq 24 \pi_{s}(T) L_{r, 1}^{x}(S) .
$$


This completes the proof of (1). The proof of (2) is now easy, since for a given $S$ with $\operatorname{rank} S=n$

$$
L_{r, 1}^{x}(S)=\sum_{k=1}^{n} k^{-1} k^{1 / r} x_{k}(S) \leq(1+\log n) L_{r, \infty}^{x}(S) .
$$

We finally remark that the preceding proposition can be used to reprove the following special (but most important) case of a deep result of Maurey [17] (for the full statement see (1.1.6)).

1.2.2. Corollary. Let $2 \leq s_{0}<s \leq \infty$ and $\frac{1}{s}+\frac{1}{r}=\frac{1}{2}$. Then $\mathscr{P}_{r, 2} \subseteq \mathscr{M}_{s_{0}, 2}$.

Proof. Define $2 \leq r<r_{0} \leq \infty$ by $1 / s_{0}+1 / r_{0}=\frac{1}{2}$. Then $\mathscr{L}_{r, \infty}^{x} \subseteq \mathscr{L}_{r_{0}, 1}^{x}$, and therefore part (1) of the proposition implies $\mathscr{P}_{r, 2} \subseteq \mathscr{L}_{r_{0}, 1}^{x} \subseteq \mathscr{M}_{s_{0}, 2}$.

1.3. A tensor multiplicativity concept. A simple but striking concept is presented which is a useful tool to improve various kinds of inequalities and furnishes the foundation for many modern results in summability and eigenvalue theory.

A subset $\mathscr{A}$ of operators $S: \mathbb{K}^{n} \rightarrow \mathbb{K}^{n}$ (where $n \in \mathbb{N}$ is arbitrary) is called tensor stable if for all $S \in \mathscr{A}\left(\mathbb{K}^{n}, \mathbb{K}^{n}\right)$ the tensor product operator

$$
S \otimes S: \mathbb{K}^{n} \otimes \mathbb{K}^{n}=\mathbb{K}^{n^{2}} \rightarrow \mathbb{K}^{n^{2}}
$$

is again in $\mathscr{A}$ (identify $\mathbb{K}^{n} \otimes \mathbb{K}^{n}$ with $\mathbb{K}^{n^{2}}$ via the bijection $\varphi$ defined by $\varphi\left(e_{i} \otimes e_{j}\right):=e_{(n-1) i+j}$ for $\left.1 \leq i, j \leq n\right)$. Moreover, let $A: \mathscr{A} \rightarrow \mathbb{R}^{+}$be a function and $a \geq 0$. Then $A$ is said to be $a$-tensor supermultiplicative if for all $S \in \mathscr{A}$

$$
A(S)^{2} \leq a A(S \otimes S),
$$

whereas it is called $a$-tensor submultiplicative if

$$
A(S \otimes S) \leq a A(S)^{2},
$$

for all $S \in \mathscr{A}$. The following simple lemma is essential.

1.3.1. Lemma. Let $\mathscr{A}$ be a tensor stable set of operators $S: \mathbb{K}^{n} \rightarrow \mathbb{K}^{n}$ and $\lambda \geq$ 0 . Moreover, let $A: \mathscr{A} \rightarrow \mathbb{R}^{+}$be a-tensor supermultiplicative and $B: \mathscr{A} \rightarrow \mathbb{R}^{+}$ be b-tensor submulitplicative such that for each $\varepsilon>0$ there is $c(\varepsilon) \geq 0$ satisfying

$$
A(S) \leq c(\varepsilon) n^{\lambda+\varepsilon} B(S)
$$

for all $n \in \mathbb{N}$ and $S \in \mathscr{A}\left(\mathbb{K}^{n}, \mathbb{K}^{n}\right)$. Then for all $n \in \mathbb{N}$ and $S \in \mathscr{A}\left(\mathbb{K}^{n}, \mathbb{K}^{n}\right)$ $A(S) \leq a b n^{\lambda} B(S)$.

Proof. Fix $\varepsilon>0$. Then there is $c(\varepsilon) \geq 0$ such that for all $n \in \mathbb{N}$ and $S \in$ $\mathscr{A}\left(\mathbb{K}^{n}, \mathbb{K}^{n}\right)$

$$
A(S)^{2} \leq a A(S \otimes S) \leq a c(\varepsilon) n^{2(\lambda+\varepsilon)} B(S \otimes S) \leq a b c(\varepsilon) n^{2(\lambda+\varepsilon)} B(S)^{2},
$$

and hence

$$
A(S) \leq(a b)^{1 / 2} c(\varepsilon)^{1 / 2} n^{\lambda+\varepsilon} B(S) .
$$

By iteration of this result $\left(c(\varepsilon)\right.$ in $(*)$ can be replaced by $\left.(a b)^{1 / 2} c(\varepsilon)^{1 / 2}\right)$ we get for all $k, n \in \mathbb{N}$ and $S \in \mathscr{A}\left(\mathbb{K}^{n}, \mathbb{K}^{n}\right)$

$$
A(S) \leq(a b)^{\sum_{1}^{k} 2^{-i} c(\varepsilon)^{2^{-k}} n^{\lambda} n^{\varepsilon} B(S) .}
$$


Therefore, if for a fixed $S \in \mathscr{A}\left(\mathbb{K}^{n}, \mathbb{K}^{n}\right)$ first $k$ tends to infinity and then $\varepsilon$ tends to zero the described inequality is obtained.

The conclusion of the lemma holds in particular, if instead of $(*)$ there are constants $\lambda, c \geq 0$ such that for all $n \in \mathbb{N}$ and $S \in \mathscr{A}\left(\mathbb{K}^{n}, \mathbb{K}^{n}\right)$

$$
A(S) \leq c n^{\lambda}(1+\log n) B(S) .
$$

Moreover, we mention that the idea of using tensor product techniques to improve constants in certain inequalities goes back to Russo [25]. Recently, Pietsch [23] used similar tensor multiplicativity techniques to improve various eigenvalue estimates of operators.

Two of our main results will follow from the next lemma which is an immediate consequence of the preceding one.

1.3.2. Lemma. For $1 \leq u, v \leq \infty$ and $0<r<\infty$ let $[\mathscr{A}, A],[\mathscr{B}, B]$ be two quasi-Banach ideals such that

$$
\mathscr{L}_{r, 1}^{x}\left(l_{u}, l_{v}\right) \subseteq \mathscr{A}\left(l_{u}, l_{v}\right) \text { and } \mathscr{B}\left(l_{u}, l_{v}\right) \subseteq \mathscr{L}_{r, \infty}^{x}\left(l_{u}, l_{v}\right) .
$$

Moreover, assume that there are constants $a, b \geq 1$ with

$$
\begin{aligned}
& A(S)^{2} \leq a A\left(S \otimes S: l_{u}^{n^{2}} \rightarrow l_{v}^{n^{2}}\right), \\
& B\left(S \otimes S: l_{u}^{n^{2}} \rightarrow l_{v}^{n^{2}}\right) \leq b B(S)^{2},
\end{aligned}
$$

for all $n \in \mathbb{N}$ and $S \in \mathscr{L}\left(l_{u}^{n}, l_{v}^{n}\right)$. Then for all $n \in \mathbb{N}$ and $S \in \mathscr{L}\left(l_{u}^{n}, l_{v}^{n}\right)$ $A(S) \leq a b B(S)$.

Proof. By the closed graph theorem there are constants $c, d \geq 1$ such that for all $S \in \mathscr{L}\left(l_{u}^{n}, l_{v}^{n}\right)$

$$
A(S) \leq c L_{r, 1}^{x}(S) \leq c(1+\log n) L_{r, \infty}^{x}(S) \leq c d(1+\log n) B(S) .
$$

Now the conclusion follows, if we apply the lemma to the set of all operators $S: \mathbb{K}^{n} \rightarrow \mathbb{K}^{n}$, the a-tensor supermultiplicative function

$$
A: S \rightsquigarrow A\left(S: l_{u}^{n} \rightarrow l_{v}^{n}\right),
$$

and the $b$-tensor submultiplicative function

$$
B: S \leadsto B\left(S: l_{u}^{n} \rightarrow l_{v}^{n}\right) .
$$

Finally, we mention

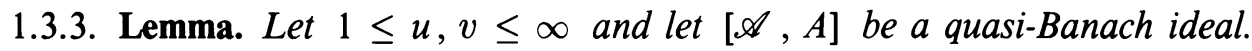
Assume that there is a constant $a \geq 1$ such that for all $n \in \mathbb{N}$

$$
A\left(\mathrm{id}: l_{u}^{n} \rightarrow l_{v}^{n}\right)^{2} \leq a A\left(\mathrm{id}: l_{u}^{n^{2}} \rightarrow l_{v}^{n^{2}}\right) .
$$

Then either

$$
\sup _{n \in \mathbb{N}} A\left(\mathrm{id}: l_{u}^{n} \rightarrow l_{v}^{n}\right) \leq a ;
$$

or there is $\varepsilon>0$ such that for large $n$

$$
n^{\varepsilon} \leq A\left(\mathrm{id}: l_{u}^{n} \rightarrow l_{v}^{n}\right) .
$$

Proof. Assume that there is $1 \neq n_{0} \in \mathbb{N}$ such that

$$
A\left(\mathrm{id}: l_{u}^{n_{0}} \rightarrow l_{v}^{n_{0}}\right)>a .
$$


Put $d:=a^{-1} A\left(\right.$ id $\left.: l_{u}^{n_{0}} \rightarrow l_{v}^{n_{0}}\right)$ and $\varepsilon:=\frac{1}{2} \log _{n_{0}} d>0$. Moreover, for $n \geq n_{0}$ choose $m \in \mathbb{N}_{0}$ such that $n_{0}^{2 m} \geq n \geq n_{0}^{m}$.

Then the conclusion follows from

$$
\begin{aligned}
A\left(\mathrm{id}: l_{u}^{n} \rightarrow l_{v}^{n}\right) & \geq A\left(\mathrm{id}: l_{u}^{n_{0}^{m}} \rightarrow l_{v}^{n_{0}^{m}}\right) \\
& \geq a d^{m}=a\left(n_{0}^{\log _{n_{0}} d}\right)^{m} \\
& =a\left(n_{0}^{2 \varepsilon}\right)^{m} \geq a n^{\varepsilon} .
\end{aligned}
$$

1.4. Tensor multiplicativity of some ideal norms. In this section we investigate tensor submultiplicativity of the operator norm and tensor supermultiplicativity of the $(s, p)$-mixing norm with respect to tensor products of $L_{p}$-spaces. In particular, we extend results of Bennett [3].

The $\varepsilon$-norm and $\pi$-norm of $z \in E \otimes F$ are denoted by

$$
\begin{aligned}
& \varepsilon(z ; E, F):=\sup \left\{|\langle z, a \otimes b\rangle| \mid a \in B_{E^{\prime}}, b \in B_{F^{\prime}}\right\}, \\
& \pi(z ; E, F):=\inf \left\{\sum_{i=1}^{n}\left\|x_{i}\right\|\left\|y_{i}\right\| \mid z=\sum_{i=1}^{n} x_{i} \otimes y_{i}\right\} .
\end{aligned}
$$

Obviously, $\varepsilon(\cdot ; E, F) \leq \pi(\cdot ; E, F)$. A norm $\alpha(\cdot ; E, F)$ on $E \otimes F$ is called reasonable, if

$$
\varepsilon(\cdot ; E, F) \leq \alpha(\cdot ; E, F) \leq \pi(\cdot ; E, F) .
$$

By $E \tilde{\otimes}_{\alpha} F$ we denote the completion of the normed space

$$
E \otimes_{\alpha} F:=(E \otimes F, \alpha(\cdot ; E, F)) .
$$

It can be seen easily that a given norm $\alpha(\cdot ; E, F)$ on $E \otimes F$ is reasonable if and only if for all $x \in E, y \in F, \alpha(x \otimes y ; E, F)=\|x\|\|y\|$, and for all $a \in E^{\prime}, b \in F^{\prime}, z \in E \otimes F,|\langle z, a \otimes b\rangle| \leq\|a\|\|b\| \alpha(z ; E, F)$.

Let us give two examples: For $1 \leq p \leq \infty$ and $z \in E \otimes F$

$$
g_{p}(z ; E, F):=\inf \left\{l_{p}\left(x_{i}\right) w_{p^{\prime}}\left(y_{i}\right) \mid z=\sum_{i=1}^{n} x_{i} \otimes y_{i}\right\},
$$

and if $T_{z} \in \mathscr{L}\left(E^{\prime}, F\right)$ denotes the operator corresponding to $z$,

$$
g_{p}^{*}(z ; E, F):=\pi_{p^{\prime}}\left(T_{z}: E^{\prime} \rightarrow F\right) .
$$

Both norms are reasonable and were intensivly studied by Chevet, Cohen, and Saphar (see e.g. [6, 7, 26]). One has

$$
g_{p^{\prime}}^{*}(z ; E, F)=\pi_{p}\left(T_{z}: E^{\prime} \rightarrow F\right) \leq N_{p}\left(T_{z}: E^{\prime} \rightarrow F\right) \leq g_{p}(z ; E, F),
$$

and equality for $p=2$. Moreover, for $1 \leq p \leq q \leq \infty$

$$
\begin{aligned}
& g_{q}(z ; E, F) \leq g_{p}(z ; E, F), \\
& g_{p}^{*}(z ; E, F) \leq g_{q}^{*}(z ; E, F) .
\end{aligned}
$$

Obviously $\alpha=g_{p}$ (resp. $\alpha=g_{p}^{*}$ ) satisfies the metric mapping property: for $T \in \mathscr{L}(E, X)$ and $S \in \mathscr{L}(F, Y)$ 
whereas for two arbitrary reasonable norms $\alpha(\cdot ; E, F)$ and $\beta(\cdot ; E, F)$ just the following estimate holds:

$$
\begin{aligned}
\|S\|\|T\| & =\sup \{\beta(S x \otimes T y ; X, Y) \mid \alpha(x \otimes y ; E, F) \leq 1\} \\
& \leq \sup \{\beta(S \otimes T(z) ; X, Y) \mid \alpha(z ; E, F) \leq 1\} \\
& =\left\|S \otimes T: E \otimes_{\alpha} F \rightarrow X \otimes_{\beta} Y\right\|
\end{aligned}
$$

(provided $S \otimes T: E \otimes_{\alpha} F \rightarrow X \otimes_{\beta} Y$ is continuous). For special norms we prove

1.4.1. Proposition. For $1 \leq u, v \leq \infty$ let $\alpha(\cdot ; E, F) \geq g_{u}(\cdot ; E, F)$ and $\beta(\cdot ; X, Y) \leq g_{v^{\prime}}^{*}(\cdot ; X, Y)$ be reasonable norms. Moreover, let $S \in \mathscr{L}(E, X)$ and $T \in \mathscr{L}(F, Y)$.

(1) If $1 \leq u \leq v \leq \infty$ then

$$
\left\|S \otimes T: E \otimes_{\alpha} F \rightarrow X \otimes_{\beta} Y\right\| \leq\|S\|\|T\| .
$$

(2) In each of the three cases

(a) $2=v \leq u \leq \infty$ and $Y$ has cotype 2,

(b) $1 \leq v \leq u=2$ and $E^{\prime}$ has cotype 2,

(c) $1 \leq v<2<u \leq \infty$ and $E^{\prime}, Y$ have cotype 2

one has

$$
\left\|S \otimes T: E \otimes_{\alpha} F \rightarrow X \otimes_{\beta} Y\right\| \leq \mu_{2, v}\left(E^{\prime}\right) \mu_{2, u^{\prime}}(Y)\|S\|\|T\| .
$$

Proof. Obviously it is sufficient to prove the assertion just for $\alpha=g_{u}$ and $\beta=g_{v^{\prime}}^{*}$. Statement (1) follows by

$$
\begin{aligned}
g_{v^{\prime}}^{*}(S \otimes T(z) ; X, Y) & \leq g_{u^{\prime}}^{*}(S \otimes T(z) ; X, Y) \\
& \leq g_{u}(S \otimes T(z) ; X, Y) \\
& \leq\|S\|\|T\| g_{u}(z ; E, F) .
\end{aligned}
$$

For the proof of $(2)(a)$ consider the commutative diagram

$$
\begin{array}{ccc}
E \otimes_{g_{u}} F & \stackrel{S \otimes T}{\rightarrow} & X \otimes_{g_{2}^{*}} Y \\
\downarrow \text { id } \otimes T & & \uparrow S \otimes \text { id } \\
E \otimes_{g_{2}} Y & = & E \otimes_{g_{2}^{*}} Y .
\end{array}
$$

Then by $\left(1.1 .4^{\prime}\right)$ and $(1.1 .7)$

$$
\begin{aligned}
\| S \otimes & T: E \otimes_{g_{u}} F \rightarrow X \otimes_{g_{2}^{*}} Y \| \\
& \leq\|S\|\left\|\operatorname{id} \otimes T: E \otimes_{g_{u}} F \rightarrow E \otimes_{g_{2}} Y\right\| \\
& =\|S\| \sup \left\{g_{2}(\operatorname{id} \otimes T(z) ; E, Y) \mid g_{u}(z ; E, F) \leq 1\right\} \\
& \leq\|S\| \sup \left\{N_{2}\left(T R: E^{\prime} \rightarrow Y\right) \mid N_{u}\left(R: E^{\prime} \rightarrow F\right) \leq 1\right\} \\
& \leq\|S\| \mu_{2, u^{\prime}}(T: F \rightarrow Y) \leq \mu_{2, u^{\prime}}(Y)\|S\|\|T\| .
\end{aligned}
$$

Dually: Under the assumption of (b) by $\left(1.1 .3^{\prime}\right)$ and (1.1.7)

$$
\begin{aligned}
\| S \otimes & T: E \otimes_{g_{2}} F \rightarrow X \otimes_{g_{v^{\prime}}^{*}} Y \| \\
& \leq\|T\|\left\|S \otimes \mathrm{id}: E \otimes_{g_{2}^{*}} Y \rightarrow X \otimes_{g_{v^{\prime}}^{*}} Y\right\| \\
& =\|T\| \sup \left\{g_{v^{\prime}}^{*}(S \otimes \operatorname{id}(z) ; X, Y) \mid g_{2}^{*}(z ; E, Y) \leq 1\right\} \\
& \leq\|T\| \sup \left\{\pi_{v}\left(R S^{\prime}: X^{\prime} \rightarrow Y\right) \mid \pi_{2}\left(R: E^{\prime} \rightarrow Y\right) \leq 1\right\} \\
& \leq\|T\| \mu_{2, v}\left(S^{\prime}: X^{\prime} \rightarrow E^{\prime}\right) \leq \mu_{2, v}\left(E^{\prime}\right)\|S\|\|T\| .
\end{aligned}
$$


Finally, assume that $1 \leq v<2<u \leq \infty$ and that $E^{\prime}, Y$ have cotype 2 . Then the diagram

$$
\begin{array}{ccc}
E \otimes_{g_{u}} F & \stackrel{S \otimes T}{\longrightarrow} & X \otimes_{g_{v^{\prime}}^{*}} Y \\
\downarrow \text { id } \otimes T & & \uparrow S \otimes \text { id } \\
E \otimes_{g_{2}^{*}} Y & = & E \otimes_{g_{2}} Y,
\end{array}
$$

commutes, and hence the assertion is an immediate consequence of $(2)(\mathrm{a})$ and (2)(b).

Let $L_{p}=L_{p}(\Omega, \Sigma, \mu)$ be an $L_{p}$-space $(1 \leq p \leq \infty)$. For $z=\sum_{i=1}^{n} x_{i} \otimes g_{i} \in$ $E \otimes L_{p}$ we define

$$
\Delta_{p}\left(z ; E, L_{p}\right):=\left(\int\left\|\sum_{i} x_{i} \otimes g_{i}\right\|^{p} d \mu\right)^{1 / p}
$$

and for $z=\sum_{i=1}^{n} f_{i} \otimes y_{i} \in L_{p} \otimes F$

$$
\Delta_{p}\left(z ; L_{p}, F\right):=\Delta_{p}\left(\sum_{i} y_{i} \otimes f_{i} ; F, L_{p}\right)
$$

(with the usual modifications for $p=\infty)$. Then $\Delta_{p}\left(\cdot ; E, L_{p}\right)$ and $\Delta_{p}\left(\cdot ; L_{p}, F\right)$ are reasonable norms and it is well known that (see e.g. $[19,27])$

$$
\begin{aligned}
g_{p}\left(\cdot ; E, L_{p}\right) & \leq \Delta_{p}\left(\cdot ; E, L_{p}\right), \\
\Delta_{p}\left(\cdot ; L_{p}, F\right) & \leq g_{p^{\prime}}^{*}\left(\cdot ; L_{p}, F\right), \\
g_{1}\left(\cdot ; E, L_{1}\right) & =\Delta_{1}\left(\cdot ; E, L_{1}\right)=\pi\left(\cdot ; E, L_{1}\right), \\
\Delta_{\infty}\left(\cdot ; L_{\infty}, F\right) & =g_{1}^{*}\left(\cdot ; L_{\infty}, F\right)=\varepsilon\left(\cdot ; L_{\infty}, F\right) .
\end{aligned}
$$

Hence the preceding proposition has the following corollary which is a proper extension of a result due to Bennett [3].

1.4.2. Corollary. Let $S \in \mathscr{L}\left(E, L_{v}\right)$ and $T \in \mathscr{L}\left(L_{u}, F\right)$.

(1) If $1 \leq u \leq v \leq \infty$, then

$$
\left\|S \otimes T: E \otimes_{\Delta_{u}} L_{u} \rightarrow L_{v} \otimes_{\Delta_{v}} F\right\| \leq\|S\|\|T\| .
$$

(2) In each of the three cases
(a) $2=v \leq u \leq \infty$ and $F$ has cotype 2,
(b) $1 \leq v \leq u=2$ and $E^{\prime}$ has cotype 2,
(c) $1 \leq v<2<u \leq \infty$ and $E^{\prime}, F$ have cotype 2

one has

$$
\left\|S \otimes T: E \otimes_{\Delta_{u}} L_{u} \rightarrow L_{v} \otimes_{\Delta_{v}} F\right\| \leq \mu_{2, v}\left(E^{\prime}\right) \mu_{2, u^{\prime}}(F)\|S\|\|T\| .
$$

We remark that this result (in contrast to Bennett's) still includes a variant of Grothendieck's Theorem namely:

$$
\begin{aligned}
\pi_{2}\left(S: l_{\infty} \rightarrow l_{1}\right) & =\left\|\mathrm{id} \otimes S: l_{2} \otimes_{\varepsilon} l_{\infty} \rightarrow l_{2} \otimes_{\Delta_{2}} l_{1}\right\| \\
& =\left\|\mathrm{id} \otimes S: l_{2} \otimes_{\Delta_{\infty}} l_{\infty} \rightarrow l_{2} \otimes_{\Delta_{2}} l_{1}\right\| \\
& \leq \mu_{2,1}\left(l_{1}\right)\|S\| .
\end{aligned}
$$


It was already noted by Bennett [3] that in the cases $1 \leq v<u<2$ and $2<v<u \leq \infty$ an inequality of the form

$$
\left\|S \otimes S: l_{u}^{n^{2}} \rightarrow l_{v}^{n^{2}}\right\| \leq c(u, v)\|S\|^{2}
$$

does not hold, where $c(u, v)$ is a constant just depending on $u$ and $v$ (see also [22, 22.4.13, second remark]).

We now formulate another special case of 1.4.1.

1.4.3. Corollary. Let $\alpha(\cdot ; E, F) \leq g_{u^{\prime}}^{*}(\cdot ; E, F)$ be a reasonable norm, $x_{1}, \ldots$, $x_{n} \in E$ and $y_{1}, \ldots, y_{n} \in F$.

(1) If $1 \leq p^{\prime} \leq u \leq \infty$, then

$$
w_{p}\left(x_{i} \otimes y_{j} ; E \tilde{\otimes}_{\alpha} F\right) \leq w_{p}\left(x_{i}\right) w_{p}\left(y_{j}\right) .
$$

(2) In each of the two cases

(a) $1 \leq u \leq p^{\prime}=2$,

(b) $1 \leq u \leq 2<p^{\prime} \leq \infty$ and $F$ has cotype 2

one has

$$
w_{p}\left(x_{i} \otimes y_{j} ; E \tilde{\otimes}_{\alpha} F\right) \leq \mu_{2, u}\left(l_{p}\right) \mu_{2, p}(F) w_{p}\left(x_{i}\right) w_{p}\left(y_{j}\right)
$$

Proof. Define

$$
S:=\sum_{i} e_{i} \otimes x_{i} \in \mathscr{L}\left(l_{p^{\prime}}^{n}, E\right), \quad T:=\sum_{j} e_{j} \otimes y_{j} \in \mathscr{L}\left(l_{p^{\prime}}^{n}, F\right) .
$$

Then

$$
\begin{gathered}
\|S\|=w_{p}\left(x_{i}\right), \quad\|T\|=w_{p}\left(y_{j}\right), \\
\left\|S \otimes T: l_{p^{\prime}}^{n^{2}} \rightarrow E \otimes_{\alpha} F\right\|=w_{p}\left(x_{i} \otimes y_{j} ; E \tilde{\otimes}_{\alpha} F\right) .
\end{gathered}
$$

Hence the conclusions follow by 1.4 .1 (replace $u$ by $p^{\prime}, \alpha$ by $\Delta_{p^{\prime}}$ and $\beta$ by $\alpha)$.

We are now ready to study tensor supermultiplicativity of the $(s, p)$-mixing norm. The following proposition and its corollary will play a crucial role for the proofs of our applications. First we set up the following notation: If $S \in$ $\mathscr{L}(E, X)$ and $T \in \mathscr{L}(F, Y)$ are two operators of finite rank, then $S \otimes T$ : $E \otimes_{\alpha} F \rightarrow X \otimes_{\beta} Y$, where $\alpha(\cdot ; E, F)$ and $\beta(\cdot ; X, Y)$ are reasonable norms, is again a continuous operator of finite rank. Hence it has a continuous extension $S \tilde{\otimes} T: E \tilde{\otimes}_{\alpha} F \rightarrow X \tilde{\otimes}_{\beta} Y$.

1.4.4. Proposition. Let $\alpha(\cdot ; E, F) \leq g_{u^{\prime}}^{*}(\cdot ; E, F)$ and $\beta(\cdot ; X, Y)$ be reasonable norms. Moreover, let $S \in \mathscr{L}(E, X)$ and $T \in \mathscr{L}(F, Y)$ be operators of finite rank.

(1) If $1 \leq p^{\prime} \leq u \leq \infty$, then

$$
\mu_{s, p}(S) \mu_{s, p}(T) \leq \mu_{s, p}\left(S \tilde{\otimes} T: E \tilde{\otimes}_{\alpha} F \rightarrow X \tilde{\otimes}_{\beta} Y\right) .
$$

(2) In each of the two cases

(a) $1 \leq u \leq p^{\prime}=2$,

(b) $1 \leq u \leq 2<p^{\prime} \leq \infty$ and $F$ has cotype 2 
one has

$$
\mu_{s, p}(S) \mu_{s, p}(T) \leq \mu_{2, u}\left(l_{p}\right) \mu_{2, p}(F) \mu_{s, p}\left(S \tilde{\otimes} T: E \tilde{\otimes}_{\alpha} F \rightarrow X \tilde{\otimes}_{\beta} Y\right) .
$$

Proof. Fix $x_{1}, \ldots, x_{m} \in E, y_{1}, \ldots, y_{m} \in F, a_{1}, \ldots, a_{m} \in X^{\prime}$, and $b_{1}, \ldots$, $b_{m} \in Y^{\prime}$. Then by (1.1.2)

$$
\begin{gathered}
\left(\sum_{i=1}^{m}\left(\sum_{k=1}^{m}\left|\left\langle S x_{i}, a_{k}\right\rangle\right|^{s}\right)^{p / s}\right)^{1 / p}\left(\sum_{j=1}^{m}\left(\sum_{l=1}^{m}\left|\left\langle T y_{j}, b_{l}\right\rangle\right|^{s}\right)^{p / s}\right)^{1 / p} \\
=\left(\sum_{i, j}\left(\sum_{k, l}\left|\left\langle S \tilde{\otimes} T\left(x_{i} \otimes y_{j}\right), a_{k} \otimes b_{l}\right\rangle\right|^{s}\right)^{p / s}\right)^{1 / p} \\
\leq \mu_{s, p}\left(S \tilde{\otimes} T: E \tilde{\otimes}_{\alpha} F \rightarrow X \tilde{\otimes}_{\beta} Y\right) \\
\cdot w_{p}\left(x_{i} \otimes y_{j} ; E \tilde{\otimes}_{\alpha} F\right) l_{s}\left(a_{k} \otimes b_{l} ;\left(X \tilde{\otimes}_{\beta} Y\right)^{\prime}\right)
\end{gathered}
$$

and since $\beta$ is a reasonable norm on $X \otimes Y$

$$
l_{s}\left(a_{k} \otimes b_{l} ;\left(X \tilde{\otimes}_{\beta} Y\right)^{\prime}\right)=l_{s}\left(a_{k}\right) l_{s}\left(b_{l}\right) \text {. }
$$

Hence both assertions follow from the preceding corollary.

Exactly in the same way it can be shown that

$$
\pi_{r, p}(S) \pi_{r, p}(T) \leq \pi_{r, p}\left(S \tilde{\otimes} T: E \tilde{\otimes}_{\alpha} F \rightarrow X \tilde{\otimes}_{\beta} Y\right),
$$

for $1 \leq p^{\prime} \leq u \leq \infty$, and

$$
\pi_{r, p}(S) \pi_{r, p}(T) \leq \mu_{2, u}\left(l_{p}\right) \mu_{2, p}(F) \pi_{r, p}\left(S \tilde{\otimes} T: E \tilde{\otimes}_{\alpha} F \rightarrow X \tilde{\otimes}_{\beta} Y\right),
$$

for $1 \leq u \leq p^{\prime}=2$ or $1 \leq u \leq 2<p^{\prime} \leq \infty$, provided $F$ has cotype 2 .

1.4.5. Corollary. Let $S \in \mathscr{L}\left(l_{u}^{n}, X\right), T \in \mathscr{L}\left(l_{u}^{n}, Y\right)$ and let $\beta$ be a reasonable norm on $X \otimes Y$.

(1) If $1 \leq p^{\prime} \leq u \leq \infty$, then

$$
\mu_{s, p}(S) \mu_{s, p}(T) \leq \mu_{s, p}\left(S \tilde{\otimes} T: l_{u}^{n^{2}} \rightarrow X \tilde{\otimes}_{\beta} Y\right) .
$$

(2) If $1 \leq u \leq 2 \leq p^{\prime} \leq \infty$, then

$$
\mu_{s, p}(S) \mu_{s, p}(T) \leq \mu_{2, u}\left(l_{p}\right) \mu_{2, p}\left(l_{u}\right) \mu_{s, p}\left(S \tilde{\otimes} T: l_{u}^{n^{2}} \rightarrow X \tilde{\otimes}_{\beta} Y\right) .
$$

Since the case $p=2$ is the most important for applications we once more refer to the upper estimates of $\mu_{2, u}\left(l_{2}\right)$ for $1 \leq u \leq 2$, mentioned at the end of 1.1 .

\section{INTEGRAL CHARACTERIZATIONS OF OPERATOR IN $L_{p}$ SPACES}

Now we are ready to prove our main results: integral characterizations of

- operators from $L_{1}$-spaces into $L_{v}$-spaces,

- Schatten-von Neumann operators,

- identity operators from $l_{u}$ into $l_{v}$. 
2.1. Operators from $L_{1}$-spaces into $L_{v}$-spaces. The following important fact was discovered by Kwapien [12] (see also [3]).

Let $1 \leq v \leq \infty, 0<p \leq 2$ and $1 \leq p \leq r \leq \infty$. If $\frac{1}{r} \leq \frac{1}{p}-\left|\frac{1}{2}-\frac{1}{v}\right|$ then

$$
\mathscr{L}\left(l_{1}, l_{v}\right)=\mathscr{P}_{r, p}\left(l_{1}, l_{v}\right)
$$

Moreover, this result is best possible in the sense that if $r$ fails to satisfy the inequality $\frac{1}{r} \leq \frac{1}{p}-\left|\frac{1}{2}-\frac{1}{v}\right|$ then $\mathscr{L}\left(l_{1}, l_{v}\right) \neq \mathscr{P}_{r, p}\left(l_{1}, l_{v}\right)$.

By use of an inclusion formula for absolutely $(r, p)$-summing operators (mentioned in the preliminary section) the proof can be restricted to the case $p=1$. In order to prove this case Kwapien interpolates between the points $v=1,2$, and $\infty$. The cases $p=1, v=1$ and $p=1, v=\infty$ are consequences of a result of Orlicz [18], which states that the identity operator on $l_{1}$ is absolutely $(2,1)$-summing, whereas the case $p=1, v=2$ obviously is Grothendieck's Theorem [9].

We now state a proper extension of Kwapien's result.

2.1.1. Theorem. Let $1 \leq v \leq \infty$ and $0<p \leq 2 \leq s \leq \infty$ such that $\frac{1}{s}=\left|\frac{1}{2}-\frac{1}{v}\right|$. Then

$$
\mathscr{L}\left(l_{1}, l_{v}\right)=\mathscr{M}_{s, p}\left(l_{1}, l_{v}\right),
$$

and for all $S \in \mathscr{L}\left(l_{1}, l_{v}\right)$

$$
\mu_{s, p}(S) \leq k(p)\|S\|,
$$

where $k(p):=\mu_{2, p}\left(l_{1}\right) \mu_{2,1}\left(l_{2}\right)$. In other words, for every $S \in \mathscr{L}\left(l_{1}, l_{v}\right)$ and every probability measure $\mu \in W\left(B_{l_{v^{\prime}}}\right)$ there is a probability measure $\nu \in W\left(B_{l_{\infty}}\right)$ such that for all $x \in l_{1}$

$$
\left(\int|\langle S x, a\rangle|^{s} d \mu(a)\right)^{1 / s} \leq k(p)\left(\int|\langle x, a\rangle|^{p} d \nu(a)\right)^{1 / p}
$$

(with the obvious modifications if $v=\infty$ or $s=\infty$ ).

Proof. Since

$$
\left[\mathscr{M}_{s, 2} \cdot \mathscr{M}_{2, p}, \mu_{s, 2} \cdot \mu_{2, p}\right] \subseteq\left[\mathscr{M}_{s, p}, \mu_{s, p}\right]
$$

and $\operatorname{id}_{l_{1}} \in \mathscr{M}_{2, p}$ for $0<p<2$ (see 1.1.1 and 1.1.7), it suffices to prove the assertion for $p=2$. Define $2 \leq r \leq \infty$ by $\frac{1}{s}+\frac{1}{r}=\frac{1}{2}$. By 1.2.1 Proposition we know $\mathscr{L}_{r, 1}^{x}\left(l_{1}, l_{v}\right) \subseteq \mathscr{M}_{s, 2}\left(l_{1}, l_{v}\right)$, and by Kwapien's theorem $\mathscr{L}\left(l_{1}, l_{v}\right)=$ $\mathscr{P}_{r, 2}\left(l_{1}, l_{v}\right) \subseteq \mathscr{L}_{r, \infty}^{x}\left(l_{1}, l_{v}\right)$.

Moreover, by $1.4 .5(2)$ and $1.4 .2(1)$ for all $S \in \mathscr{L}\left(l_{1}^{n}, l_{v}^{n}\right)$

$$
\begin{gathered}
\mu_{s, 2}(S)^{2} \leq k(2) \mu_{s, 2}\left(S \otimes S: l_{1}^{n^{2}} \rightarrow l_{v}^{n^{2}}\right), \\
\left\|S \otimes S: l_{1}^{n^{2}} \rightarrow l_{v}^{n^{2}}\right\| \leq\|S\|^{2} .
\end{gathered}
$$

Hence 1.3.2 Lemma implies that for all $S \in \mathscr{L}\left(l_{1}^{n}, l_{v}^{n}\right) \mu_{s, 2}(S) \leq k(2)\|S\|$.

Let now $S \in \mathscr{L}\left(l_{1}, l_{v}\right)$. We use the local definition (1.1.2) in order to show that $S$ is $(s, 2)$-mixing. Fix $x_{1}, \ldots, x_{m} \in l_{1}$ and $b_{1}, \ldots, b_{m} \in l_{v^{\prime}}$ (for 
$v=\infty$ the assertion is trivial). For $n \in \mathbb{N}$ define the operators

$$
\begin{aligned}
S_{n}: & l_{1}^{n} \rightarrow l_{v}^{n} \\
& \left(\xi_{i}\right) \rightsquigarrow\left(S\left(\xi_{1}, \ldots, \xi_{n}, 0, \ldots\right)_{i}\right)_{i=1}^{n} \\
P_{n}: & l_{1} \rightarrow l_{1}^{n} \\
& \left(\xi_{i}\right) \rightsquigarrow\left(\xi_{1}, \ldots, \xi_{n}\right) \\
Q_{n}: & l_{v^{\prime}} \rightarrow l_{v^{\prime}}^{n} \\
& \left(\xi_{i}\right) \rightsquigarrow\left(\xi_{1}, \ldots, \xi_{n}\right) .
\end{aligned}
$$

Then for all $n \in \mathbb{N}$

$$
\begin{gathered}
\left(\sum_{i}\left(\sum_{k}\left|\left\langle S_{n} P_{n} x_{i}, Q_{n} b_{k}\right\rangle\right|^{s}\right)^{2 / s}\right)^{1 / 2} \\
\leq \mu_{s, 2}\left(S_{n}\right) w_{2}\left(P_{n} x_{i}\right) l_{s}\left(Q_{n} b_{k}\right) \\
\leq k(2)\|S\| w_{2}\left(x_{i}\right) l_{s}\left(b_{k}\right) .
\end{gathered}
$$

Since for fixed $i$ and $k \lim _{n \rightarrow \infty}\left|\left\langle S_{n} P_{n} x_{i}, Q_{n} b_{k}\right\rangle\right|=\left|\left\langle S x_{i}, b_{k}\right\rangle\right|$, we get $S \in$ $\mathscr{M}_{s, 2}\left(l_{1}, l_{v}\right)$ and $\mu_{s, 2}(S) \leq k(2)\|S\|$.

Following the local techniques of Lindenstrauss and Pelczyński [14] the theorem can be extended to operators acting between so-called $\mathscr{L}_{1, \lambda^{-}}$and $\mathscr{L}_{v, \mu^{-}}$

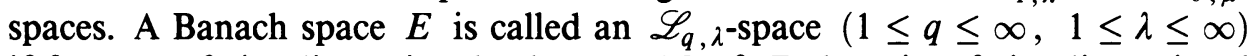
if for every finite-dimensional subspace $M$ of $E$ there is a finite-dimensional subspace $N$ of $E$ such that $M \subseteq N$ and the Banach-Mazur distance

$$
d\left(N, l_{q}^{n}\right):=\inf \left\{\|T\|\left\|T^{-1}\right\| \mid T \in \mathscr{L}\left(N, l_{q}^{n}\right) \text { bijective }\right\} \leq \lambda,
$$

where $n:=\operatorname{dim} N$. Every $L_{q}$-space is an $\mathscr{L}_{q, \lambda}$-space for all $\lambda>1$ and every

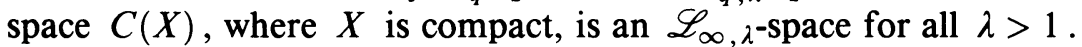

By use of 1.1.2 and standard (local) arguments the following extensions of 2.1.1 can be shown:

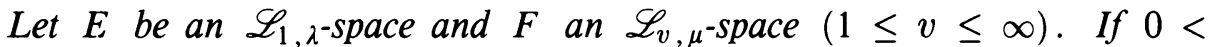
$p \leq 2 \leq s \leq \infty$ and $\frac{1}{s}=\left|\frac{1}{2}-\frac{1}{v}\right|$ then $\mathscr{L}(E, F)=\mathscr{M}_{s, p}(E, \bar{F})$, and for all $S \in \mathscr{L}(E, F), \mu_{s, p}(S) \leq k(p) \lambda \mu\|S\|$.

Finally we note some interesting composition formulas (all of which are equivalent to our theorem). For this we need some more notation. The canonical embedding of a Banach space $E$ into its bidual $E^{\prime \prime}$ will be denoted by $K_{E}$. Let $1 \leq p \leq \infty$. By definition $\mathscr{L}_{p}$ is the ideal of all operators $S \in \mathscr{L}(E, F)$ such that $\bar{K}_{F} S$ factors through an appropriate $L_{p}$-space. $S \in \mathscr{L}(E, F)$ belongs to the ideal $\mathscr{I}_{p}$ if there is a $\mu \in W\left(B_{E^{\prime}}\right)$ such that $K_{F} S$ factors through the formal identity $C\left(B_{E^{\prime}}\right) \rightarrow L_{p}(\mu)$. Moreover, we write $S \in \mathscr{P}_{p}^{\text {dual }}(E, F)$ if $S^{\prime} \in \mathscr{P}_{p}\left(F^{\prime}, E^{\prime}\right)$.

2.1.2. Corollary. Let $1 \leq v \leq \infty$ and $1 \leq p \leq 2 \leq s \leq \infty$. If $\frac{1}{s}=\left|\frac{1}{2}-\frac{1}{v}\right|$ then

(1) $\mathscr{L}_{v} \cdot \mathscr{L}_{1} \subseteq \mathscr{M}_{s, p}$,

(2) $\mathscr{P}_{s} \cdot \mathscr{L}_{v} \cdot \mathscr{L}_{1} \subseteq \mathscr{P}_{p}$,

(3) $\mathscr{L}_{v} \cdot \mathscr{L}_{1} \cdot \mathscr{I}_{p^{\prime}} \subseteq \mathscr{I}_{s^{\prime}}$,

(4) $\mathscr{L}_{v} \cdot \mathscr{L}_{1} \cdot \mathscr{N}_{p^{\prime}} \subseteq \mathscr{N}_{s^{\prime}}$,

(5) $\mathscr{L}_{1} \cdot \mathscr{I}_{p^{\prime}} \cdot \mathscr{P}_{s} \subseteq \mathscr{P}_{v}^{\text {dual }} \cdot \mathscr{P}_{v^{\prime}}$,

(6) $\mathscr{I}_{p^{\prime}} \cdot \mathscr{P}_{s} \cdot \mathscr{L}_{v} \subseteq \mathscr{P}_{1}^{\text {dual }}$. 
Sketch of the Proof. Let us first check (1). By definition for every $S \in$ $\mathscr{L}_{v} \cdot \mathscr{L}_{1}(E, F)$ there are an $L_{1}$-space $L_{1}$, an $L_{v}$-space $L_{v}$ and operators

$$
X \in \mathscr{L}\left(E^{\prime \prime}, L_{1}^{\prime \prime}\right), \quad U \in \mathscr{L}\left(L_{1}^{\prime \prime}, L_{v}^{\prime \prime}\right), \quad Y \in \mathscr{L}\left(L_{v}^{\prime \prime}, F^{\prime \prime}\right),
$$

such that the following diagram commutes:

$$
\begin{array}{lll}
E^{\prime \prime} & \stackrel{S^{\prime \prime}}{\rightarrow} & F^{\prime \prime} \\
\downarrow X & & \uparrow Y \\
L_{1}^{\prime \prime} & \stackrel{U}{\rightarrow} & L_{v}^{\prime \prime} .
\end{array}
$$

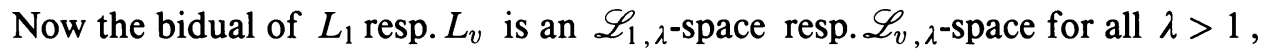
and hence $U$ and in particular $S^{\prime \prime}$ are $(s, p)$-mixing. But since $S \in \mathscr{M}_{s, p}$ if $S^{\prime \prime} \in \mathscr{M}_{s, p}$ (this is an immediate consequence of (1.1.2)), the proof of (1) is complete. The assertions (2), (3) and (4) now follow by the inclusion formulas

$$
\mathscr{P}_{s} \cdot \mathscr{M}_{s, p} \subseteq \mathscr{P}_{p}, \quad \mathscr{M}_{s, p} \cdot \mathscr{F}_{p^{\prime}} \subseteq \mathscr{I}_{s^{\prime}}, \quad \mathscr{M}_{s, p} \cdot \mathscr{N}_{p^{\prime}} \subseteq \mathscr{N}_{s^{\prime}}
$$

(see (1.1.3), (1.1.4) and [22, 20.2]). Moreover, (3) implies

$$
\mathscr{L}_{1} \cdot \mathscr{I}_{p^{\prime}} \subseteq \mathscr{L}_{v}^{-1} \cdot \mathscr{I}_{s^{\prime}}=\mathscr{L}_{v}^{*} \cdot \mathscr{P}_{s}^{-1}=\mathscr{P}_{v}^{\text {dual }} \cdot \mathscr{P}_{v^{\prime}} \cdot \mathscr{P}_{s}^{-1},
$$

where $\mathscr{L}_{v}^{*}$ denotes the adjoint ideal of $\mathscr{L}_{v}$ which by the Persson-Pietsch trace duality and a deep factorization theorem of Kwapien equals $\mathscr{P}_{v}^{\text {dual }} \cdot \mathscr{P}_{v^{\prime}}$ (see $\left[22,17.4 .3\right.$. and 19.3.10]). For the proof of the equality $\mathscr{L}_{v}^{-1} \cdot \mathscr{I}_{s^{\prime}}=\mathscr{L}_{v}^{*} \cdot \mathscr{P}_{s}^{-1}$ use e.g. the general quotient formula 4.4.2. of [8]. In a similar way (5) implies (6).

2.2. Schatten-von Neumann classes. By definition the Schatten-von Neumann classes are

$$
\mathscr{A}_{r}\left(l_{2}, l_{2}\right):=\mathscr{L}_{r}^{a}\left(l_{2}, l_{2}\right), \quad 0<r<\infty .
$$

For $S \in \mathscr{A}_{r}\left(l_{2}, l_{2}\right)$ put

$$
A_{r}(S):=L_{r}^{a}(S)=\left(\sum_{k=1}^{\infty} a_{k}(S)^{r}\right)^{1 / r} .
$$

A result of Mitjagin which was first published in [12], states that for $2 \leq r<\infty$,

$$
\left[\mathscr{A}_{r}\left(l_{2}, l_{2}\right), A_{r}\right]=\left[\mathscr{P}_{r, 2}\left(l_{2}, l_{2}\right), \pi_{r, 2}\right]
$$

(see e.g. [23, 2.11.28]). By use of our tensor multiplicative concept one can even prove the following integral characterization of the Schatten-von Neumann classes.

2.2.1. Theorem. Let $2 \leq r<\infty, 0<p \leq 2<s \leq \infty$ and $\frac{1}{s}+\frac{1}{r}=\frac{1}{2}$. Then $\mathscr{A}_{r}\left(l_{2}, l_{2}\right)=\mathscr{M}_{s, p}\left(l_{2}, l_{2}\right)$, and for every $S \in \mathscr{A}_{r}\left(l_{2}, l_{2}\right)$,

$$
A_{r}(S) \leq \mu_{s, p}(S) \leq \mu_{2, p}\left(l_{2}\right) A_{r}(S),
$$

i.e., for all probability measures $\mu \in W\left(B_{l_{2}}\right)$ there is a probability measure $\nu \in W\left(B_{l_{2}}\right)$ such that for all $x \in l_{2}$

$$
\left(\int|\langle S x, a\rangle|^{s} d \mu(a)\right)^{1 / s} \leq \mu_{2, p}\left(l_{2}\right) A_{r}(S)\left(\int|\langle x, a\rangle|^{p} d \nu(a)\right)^{1 / p} .
$$


Proof. As in the proof of 2.1.1 we may restrict our considerations to the case $p=2$. By (the easier part of) Mitjagin's result

$$
\left[\mathscr{M}_{s, 2}\left(l_{2}, l_{2}\right), \mu_{s, 2}\right] \subseteq\left[\mathscr{P}_{r, 2}\left(l_{2}, l_{2}\right), \pi_{r, 2}\right] \subseteq\left[\mathscr{A}_{r}\left(l_{2}, l_{2}\right), A_{r}\right]
$$

For the converse inclusion we again apply 1.3.2 Lemma. One has

$$
\mathscr{A}_{r}\left(l_{2}, l_{2}\right) \subseteq \mathscr{L}_{r, \infty}^{a}\left(l_{2}, l_{2}\right)=\mathscr{L}_{r, \infty}^{x}\left(l_{2}, l_{2}\right)
$$

and by $1.2 .1, \quad \mathscr{L}_{r, 1}^{x}\left(l_{2}, l_{2}\right) \subseteq \mathscr{M}_{s, 2}\left(l_{2}, l_{2}\right)$.

Moreover, 1.4.5(1) and [23, 2.11.22] imply for all $S \in \mathscr{L}\left(l_{2}^{n}, l_{2}^{n}\right)$

$$
\mu_{s, 2}(S)^{2} \leq \mu_{s, 2}\left(S \otimes S: l_{2}^{n^{2}} \rightarrow l_{2}^{n^{2}}\right) \quad A_{r}\left(S \otimes S: l_{2}^{n^{2}} \rightarrow l_{2}^{n^{2}}\right)=A_{r}(S)^{2},
$$

and hence by 1.3 .2 for all $S \in \mathscr{L}\left(l_{2}^{n}, l_{2}^{n}\right), \mu_{s, 2}(S) \leq A_{r}(S)$.

Using the final argument of the proof of 2.1.1 we get the desired inclusion

$$
\left[\mathscr{A}_{r}\left(l_{2}, l_{2}\right), A_{r}\right] \subseteq\left[\mathscr{M}_{s, 2}\left(l_{2}, l_{2}\right), \mu_{s, 2}\right] .
$$

As a corollary we mention the full statement of Mitjagin's theorem (with a slightly better norm estimate).

2.2.2. Corollary. Let $2 \leq r<\infty, 0<p \leq 2$, and $p \leq q \leq \infty$ with $\frac{1}{q}=$ $\frac{1}{r}+\frac{1}{p}-\frac{1}{2}$. Then

$$
\mathscr{A}_{r}\left(l_{2}, l_{2}\right)=\mathscr{P}_{q, p}\left(l_{2}, l_{2}\right),
$$

and for all $S \in \mathscr{A}_{r}\left(l_{2}, l_{2}\right)$

$$
A_{r}(S) \leq \pi_{q, p}(S) \leq \mu_{2, p}\left(l_{2}\right) A_{r}(S) .
$$

Proof. Since $p \leq 2, q \leq r$ and $\frac{1}{p}-\frac{1}{q}=\frac{1}{2}-\frac{1}{r}$,

$$
\left[\mathscr{P}_{q, p}\left(l_{2}, l_{2}\right), \pi_{q, p}\right] \subseteq\left[\mathscr{P}_{r, 2}\left(l_{2}, l_{2}\right), \pi_{r, 2}\right] \subseteq\left[\mathscr{A}_{r}\left(l_{2}, l_{2}\right), A_{r}\right] .
$$

Conversely: If $\frac{1}{s}+\frac{1}{q}=\frac{1}{p}$, then $\frac{1}{s}+\frac{1}{r}=\frac{1}{2}$. Hence by the preceding theorem

$$
\mathscr{A}_{r}\left(l_{2}, l_{2}\right) \subseteq \mathscr{M}_{s, p}\left(l_{2}, l_{2}\right) \subseteq \mathscr{P}_{q, p}\left(l_{2}, l_{2}\right),
$$

and for all $S \in \mathscr{A}_{r}\left(l_{2}, l_{2}\right)$

$$
\pi_{q, p}(S) \leq \mu_{s, p}(S) \leq \mu_{2, p}\left(l_{2}\right) A_{r}(S) .
$$

2.3. Identity operators from $l_{u}$ into $l_{v}$. The following characterization of those identity operators $I: l_{u} \hookrightarrow l_{v}(1 \leq u \leq v \leq \infty)$ which are absolutely $(r, 2)$ summing has been determined by Bennett [2] (see also [3]) and the first author [4].

Let $1 \leq u \leq 2,1 \leq u \leq v \leq \infty$, and $2 \leq r \leq \infty$. Then $I \in \mathscr{P}_{r, 2}\left(l_{u}, l_{v}\right)$ if $\frac{1}{r} \leq \frac{1}{u}-\max \left\{\frac{1}{v}, \frac{1}{2}\right\}$.

Again this result is sharp in the sense that $I \notin \mathscr{P}_{r, 2}\left(l_{u}, l_{v}\right)$ if $\frac{1}{r}-\frac{1}{u}+$ $\max \left\{\frac{1}{v}, \frac{1}{2}\right\}=: \varepsilon>0$. In this case for all $n \in \mathbb{N}$

$$
n^{\varepsilon} \leq \pi_{r, 2}\left(\mathrm{id}: l_{u}^{n} \rightarrow l_{v}^{n}\right)
$$

(see the proofs of [4, Theorems 1 and 2]). Especially the case $v=2$ has proved successful in its application to the theory of distribution of eigenvalues of matrices and integral operators as has been discovered by König, Pietsch, Retherford, and Tomczak-Jaegermann (see [11, 23]). 
In this section we reprove and extend this result by use of our tensor product trick within the theory of $s$-numbers. For this purpose we recall the definition of Hilbert numbers given by Bauhardt [1]. The $n$th Hilbert number of an operator $S \in \mathscr{L}(E, F)$ is defined by

$$
h_{n}(S):=\sup \left\{a_{n}(Y S X) \mid\left\|X: l_{2} \rightarrow E\right\| \leq 1,\left\|Y: F \rightarrow l_{2}\right\| \leq 1\right\} .
$$

We start with a result which is implicitly found in [21].

\subsubsection{Lemma.}

(1) Let $S \in \mathscr{L}(E, F)$ with $\operatorname{dim} E=\operatorname{dim} F=\operatorname{rank} S=n$. Then for all $1 \leq k \leq n, h_{k}(S) a_{n-k+1}\left(S^{-1}\right) \leq 1$.

(2) Let $S \in \mathscr{L}\left(E, l_{2}^{n}\right)$ with $\operatorname{dim} E=\operatorname{rank} S=n$. Then for all $1 \leq k \leq$ $n, x_{k}(S) a_{n-k+1}\left(S^{-1}\right)=1$.

Proof. (1) Let $0<\varepsilon<1$ and $1 \leq k \leq n$. By Bauhardt's characterization [1] of Hilbert numbers (see also [22, 11.4.3]) there are operators $X \in \mathscr{L}\left(l_{2}^{k}, E\right)$ and $Y \in \mathscr{L}\left(F, l_{2}^{k}\right)$ such that $\|X\| \leq 1,\|Y\| \leq 1$, and $(1-\varepsilon) h_{k}(S)$ id $=Y S X$.

Put $\rho:=(1-\varepsilon) h_{k}(S)$ and $A:=S^{-1}-\rho^{-1} X Y$. Since $A S X=0$ we have

$$
\operatorname{dim} \operatorname{kern} A \geq \operatorname{rank} S X=k>k-1
$$

(note that $S X$ is injective), and in particular

$$
\text { rank } A=n-\operatorname{dim} \operatorname{kern} A<n-k+1 .
$$

Consequently, the conslusion follows by

$$
(1-\varepsilon) h_{k}(S) a_{n-k+1}\left(S^{-1}\right) \leq \rho\left\|S^{-1}-A\right\| \leq\|X\|\|Y\| \leq 1 .
$$
hand

(2) By (1) the inequality $\leq$ is clear, since $x_{k}(S)=h_{k}(S)$. On the other

$$
\begin{aligned}
1 & =x_{n}\left(\mathrm{id}: l_{2}^{n} \rightarrow l_{2}^{n}\right) \\
& \leq x_{k}\left(S: E \rightarrow l_{2}^{n}\right) x_{n-k+1}\left(S^{-1}: l_{2}^{n} \rightarrow E\right) \\
& =x_{k}\left(S: E \rightarrow l_{2}^{n}\right) a_{n-k+1}\left(S^{-1}: l_{2}^{n} \rightarrow E\right) .
\end{aligned}
$$

As a consequence we prove

2.3.3. Proposition. Let $1 \leq u \leq 2$ and $1 \leq u \leq v \leq \infty$. Then for all $1 \leq k \leq n$

(1) $x_{k}\left(\right.$ id $\left.: l_{u}^{n} \rightarrow l_{2}^{n}\right)=k^{1 / 2-1 / u}$.

(2) $x_{k}\left(\right.$ id $\left.: l_{u}^{n} \rightarrow l_{v}^{n}\right) \leq k^{\max \{1 / v, 1 / 2\}-1 / u}$.

Proof. Since by a result of Pietsch [23, 2.9.8] for $1 \leq u \leq 2$ and $1 \leq k \leq$ $n, a_{n-k+1}\left(\right.$ id $\left.: l_{2}^{n} \rightarrow l_{u}^{n}\right)=k^{1 / u-1 / 2}$, the preceding lemma proves (1).

Let us now prove (2). If $1 \leq u \leq 2 \leq v \leq \infty$ then for all $1 \leq k \leq n$

$$
x_{k}\left(\mathrm{id}: l_{u}^{n} \rightarrow l_{v}^{n}\right) \leq x_{k}\left(\mathrm{id}: l_{u}^{n} \rightarrow l_{2}^{n}\right)=k^{1 / 2-1 / u} .
$$

The case $1 \leq u \leq v<2$ follows by interpolation: Define

$$
\theta:=\frac{1 / u-1 / v}{1 / u-1 / 2}
$$

so that $\frac{1}{v}=\frac{\theta}{2}+\frac{1-\theta}{u}$, and let $A \in \mathscr{L}\left(l_{2}, l_{u}^{n}\right)$ with $\|A\| \leq 1$. Then by Hölder's inequality for $x \in l_{2}$

$$
l_{v}(A x) \leq l_{2}(A x)^{\theta} l_{u}(A x)^{1-\theta} .
$$


Hence for each subspace $M$ of $l_{2}$ we conclude with the natural embedding $I_{M}^{l_{2}}$ from $M$ into $l_{2}$

$$
\left\|A I_{M}^{l_{2}}: M \rightarrow l_{v}^{n}\right\| \leq\left\|A I_{M}^{l_{2}}: M \rightarrow l_{2}^{n}\right\|^{\theta}\left\|A I_{M}^{l_{2}}: M \rightarrow l_{u}^{n}\right\|^{1-\theta} \leq\left\|A I_{M}^{l_{2}}: M \rightarrow l_{2}^{n}\right\|^{\theta} \text {, }
$$

and consequencely for all $1 \leq k \leq n$

$$
a_{k}\left(A: l_{2} \rightarrow l_{v}^{n}\right) \leq a_{k}\left(A: l_{2} \rightarrow l_{2}^{n}\right)^{\theta}
$$

(obviously the latter inequlaity holds for the so-called Gelfand numbers which for operators defined on $l_{2}$ coincide with the approximation numbers, see [11 or 23]). Finally, we get for all $1 \leq k \leq n$

$$
x_{k}\left(\mathrm{id}: l_{u}^{n} \rightarrow l_{v}^{n}\right) \leq x_{k}\left(\mathrm{id}: l_{u}^{n} \rightarrow l_{2}^{n}\right)^{\theta}=k^{(1 / 2-1 / u) \theta}=k^{1 / v-1 u} .
$$

We are now prepared to state the following abstract version of the characterization of absolutely $(r, 2)$-summing identity operators $I: l_{u} \hookrightarrow l_{v}$ mentioned at the begining of this section.

2.3.4. Lemma. Let $1 \leq u \leq 2,1 \leq u \leq v \leq \infty$, and $2 \leq r<\infty$. Let [AA, A] be a quasi-Banach ideal such that

$$
\mathscr{L}_{r, 1}^{x}\left(l_{u}, l_{v}\right) \subseteq \mathscr{A}\left(l_{u}, l_{v}\right) \subseteq \mathscr{L}_{r, \infty}^{x}\left(l_{u}, l_{v}\right)
$$

Moreover, assume that there is a constant $a \geq 1$ with

$$
A\left(\mathrm{id}: l_{u}^{n} \rightarrow l_{v}^{n}\right)^{2} \leq a A\left(\mathrm{id}: l_{u}^{n^{2}} \rightarrow l_{v}^{n^{2}}\right),
$$

for all $n \in \mathbb{N}$. Then the following alternative holds:

(1) If $\frac{1}{r} \leq \frac{1}{u}-\max \left\{\frac{1}{v}, \frac{1}{2}\right\}$, then $\sup _{n \in \mathbb{N}} A\left(\mathrm{id}: l_{u}^{n} \rightarrow l_{v}^{n}\right) \leq a$.

(2) If $\frac{1}{r}-\frac{1}{u}+\max \left\{\frac{1}{v}, \frac{1}{2}\right\}=: \varepsilon>0$, then $n^{\varepsilon} \leq A\left(\mathrm{id}: l_{u}^{n} \rightarrow l_{v}^{n}\right)$ for large $n$.

Proof. (1) We denote by $\mathscr{C}$ the tensor stable set for all identity operators id $: \mathbb{K}^{n} \rightarrow \mathbb{K}^{n}$, where $n \in \mathbb{N}$. Then by assumption $A:$ id $\leadsto A\left(\mathrm{id}: l_{u}^{n} \rightarrow l_{v}^{n}\right)$ is an $a$-tensor supermultiplicative function on $\mathscr{C}$ and

$$
\|\cdot\|: \mathrm{id} \rightsquigarrow\left\|\mathrm{id}: l_{u}^{n} \rightarrow l_{v}^{n}\right\|
$$

is obviously a 1-tensor submultiplicative function on $\mathscr{C}$. Moreover, by the closed graph theorem and the preceding proposition there is a constant $c \geq 1$ such that for all $n \in \mathbb{N}$

$$
\begin{aligned}
A\left(\mathrm{id}: l_{u}^{n} \rightarrow l_{v}^{n}\right) & \leq c L_{r, 1}^{x}\left(\mathrm{id}: l_{u}^{n} \rightarrow l_{v}^{n}\right) \\
& =c \sum_{k=1}^{n} k^{1 / r-1} x_{k}\left(\mathrm{id}: l_{u}^{n} \rightarrow l_{v}^{n}\right) \\
& =c \sum_{k=1}^{n} k^{1 / r-1+\max \{1 / v, 1 / 2\}-1 / u} \\
& \leq c(1+\log n)\left\|\mathrm{id}: l_{u}^{n} \rightarrow l_{v}^{n}\right\| .
\end{aligned}
$$

But then the desired inequality follows from 1.3.1.

(2) There is $\delta_{0}>0$ such that for all $0<\delta<\delta_{0}$

$$
\varepsilon_{\delta}:=\frac{1}{r+\delta}-\frac{1}{u}+\max \left\{\frac{1}{v}, \frac{1}{2}\right\}>0
$$


Moreover, there is a (uniform) constant $c \geq 1$ such that for all $0<\delta<\delta_{o}$ and $n \in \mathbb{N}$

$$
\pi_{r+\delta, 2}\left(\mathrm{id}: l_{u}^{n} \rightarrow l_{v}^{n}\right) \leq c L_{r+\delta, 1}^{x}\left(\mathrm{id}: l_{u}^{n} \rightarrow l_{v}^{n}\right)
$$

(see e.g. the proofs of $[11,1$. d.20 and 2.a.11]). Hence by assumption and (2.3.1) there is $d \geq 1$ such that for all $0<\delta<\delta_{o}$ and $n \in \mathbb{N}$

$$
\begin{aligned}
n^{\varepsilon_{\delta}} & \leq c L_{r+\delta, 1}^{x}\left(\mathrm{id}: l_{u}^{n} \rightarrow l_{v}^{n}\right) \\
& \leq c L_{r, \infty}^{x}\left(\mathrm{id}: l_{u}^{n} \rightarrow l_{v}^{n}\right) \leq c d A\left(\mathrm{id}: l_{u}^{n} \rightarrow l_{v}^{n}\right) .
\end{aligned}
$$

Finally if $\delta$ tends to 0 for each fixed $n$, we obtain (2).

An almost immediate consequence of this lemma is the following main result of this section.

2.3.5. Theorem. Let $1 \leq u \leq 2,1 \leq u \leq v \leq \infty$ and $0<p \leq 2 \leq s \leq \infty$.

(1) If $\frac{1}{s} \geq \frac{1}{2}-\frac{1}{u}+\max \left\{\frac{1}{v}, \frac{1}{2}\right\}$, then $I \in \mathscr{M}_{s, p}\left(l_{u}, l_{v}\right)$ and

$$
\mu_{s, p}\left(I: l_{u} \hookrightarrow l_{v}\right) \leq \mu_{2, p}\left(l_{u}\right) \mu_{2, u}\left(l_{2}\right) .
$$

(2) If $\frac{1}{2}-\frac{1}{u}+\max \left\{\frac{1}{v}, \frac{1}{2}\right\}-\frac{1}{s}=: \varepsilon>0$, then $I \notin \mathscr{M}_{s, p}\left(l_{u}, l_{v}\right)$ and

$$
n^{\varepsilon} \leq \mu_{s, p}\left(\mathrm{id}: l_{u}^{n} \rightarrow l_{v}^{n}\right), \quad \text { for all } n \in \mathbb{N} .
$$

Proof. In order to prove (1) we first observe the following. Exactly as in the proof of Theorem 2.1.1 it is enough to show that in the case $p=2$ for all $n \in \mathbb{N}, \mu_{s, 2}\left(\mathrm{id}: l_{u}^{n} \rightarrow l_{v}^{n}\right) \leq \mu_{2, u}\left(l_{2}\right)$.

Since by 1.2 .1

$$
\mathscr{L}_{r, 1}^{x} \subseteq \mathscr{M}_{s, 2} \subseteq \mathscr{L}_{r, \infty}^{x}, \quad \frac{1}{s}+\frac{1}{r}=\frac{1}{2},
$$

and by $1.4 .5(2)$ for all $n \in \mathbb{N}$

$$
\mu_{s, 2}\left(\mathrm{id}: l_{u}^{n} \rightarrow l_{v}^{n}\right)^{2} \leq \mu_{2, u}\left(l_{2}\right) \mu_{s, 2}\left(\mathrm{id}: l_{u}^{n^{2}} \rightarrow l_{v}^{n^{2}}\right),
$$

we just apply the preceding lemma.

Finally we prove (2). By (2.3.1) we know that for all $n \in \mathbb{N}$

$$
n^{\varepsilon} \leq \pi_{r, 2}\left(\mathrm{id}: l_{u}^{n} \rightarrow l_{v}^{n}\right) \leq \mu_{s, 2}\left(\mathrm{id}: l_{u}^{n} \rightarrow l_{v}^{n}\right), \quad \frac{1}{s}+\frac{1}{r}=\frac{1}{2} .
$$

Since $\left[\mathscr{M}_{s, p}, \mu_{s, p}\right] \subseteq\left[\mathscr{M}_{s, 2}, \mu_{s, 2}\right]$ this implies the desired result.

Let us again give a second more analytic formulation of part (1): If $1 \leq$ $u \leq 2,1 \leq u \leq v<\infty, 0<p \leq 2 \leq s<\infty$, and $\frac{1}{s} \geq \frac{1}{2}-\frac{1}{u}+\max \left\{\frac{1}{v}, \frac{1}{2}\right\}$, then for every probability measure $\mu \in W\left(B_{l_{v^{\prime}}}\right)$ there is a probability measure $\nu \in W\left(B_{l_{u^{\prime}}}\right)$ such that for all $x \in l_{u}$

$$
\left(\int|\langle x, a\rangle|^{s} d \mu(a)\right)^{1 / s} \leq \mu_{2, p}\left(l_{u}\right) \mu_{2, u}\left(l_{2}\right)\left(\int|\langle x, a\rangle|^{p} d \nu(a)\right)^{1 / p} .
$$

In particular, we reproved the characterization of absolutely $(r, 2)$-summing identity operators $I: l_{u} \hookrightarrow l_{v}$ (with new estimates for the norms). The full statement is 
2.3.6. Corollary. Let $1 \leq u \leq 2,1 \leq u \leq v \leq \infty, 0<p \leq 2$, and $0<p \leq$ $r \leq \infty$.

(1) If $\frac{1}{r} \leq \frac{1}{p}-\frac{1}{2}+\frac{1}{u}-\max \left\{\frac{1}{v}, \frac{1}{2}\right\}$, then $I \in \mathscr{P}_{r, p}\left(l_{u}, l_{v}\right)$ and $\pi_{r, p}(I) \leq$ $\mu_{2, p}\left(l_{u}\right) \mu_{2, u}\left(l_{2}\right)$.

(2) If $\frac{1}{r}-\frac{1}{p}+\frac{1}{2}-\frac{1}{u}+\max \left\{\frac{1}{v}, \frac{1}{2}\right\}=: \varepsilon>0$, then $I \notin \mathscr{P}_{r, p}\left(l_{u}, l_{v}\right)$ and $n^{\varepsilon} \leq \pi_{r, p}\left(\mathrm{id}: l_{u}^{n} \rightarrow l_{v}^{n}\right)$ for all $n \in \mathbb{N}$.

Proof. Since $\left[\mathscr{M}_{s, p}, \mu_{s, p}\right] \subseteq\left[\mathscr{P}_{r, p}, \pi_{r, p}\right]$ for $\frac{1}{s}+\frac{1}{r}=\frac{1}{p}$, statement (1) is a consequence of part (1) of the theorem. Part (2) is again an easy consequence of (2.3.1).

We finish this section with the following remark:

For $1 \leq p, q \leq \infty$ let $A$ be a continuous bilinear form on $l_{p} \times l_{q}$. Then

$$
\left(\sum_{i=1}^{\infty}\left(\sum_{j=1}^{\infty}\left|A\left(e_{i}, e_{j}\right)\right|^{v}\right)^{r / v}\right)^{1 / r} \leq \mu_{2, p^{\prime}}\left(l_{q^{\prime}}\right) \mu_{2, q^{\prime}}\left(l_{2}\right)\|A\|,
$$

if $1 \leq p^{\prime}, q^{\prime} \leq 2,1 \leq p^{\prime} \leq r \leq \infty, 1 \leq q^{\prime} \leq v \leq \infty$ and $\frac{1}{r}+\max \left\{\frac{1}{v}, \frac{1}{2}\right\}=$ $\frac{3}{2}-\frac{1}{p}-\frac{1}{q}$.

This comprises and extends the main results of Hardy and Littlewood's paper [10]. By the last corollary the proof is easy: if $\hat{A}: l_{p} \rightarrow l_{q^{\prime}}$ is the linear operator corresponding to the bilinear form $A$, then $I \hat{A}: l_{p} \rightarrow l_{q^{\prime}} \hookrightarrow l_{v}$ is absolutely $\left(r, p^{\prime}\right)$-summing and

$$
\pi_{r, p^{\prime}}(I \hat{A}) \leq \mu_{2, p^{\prime}}\left(l_{q^{\prime}}\right) \mu_{2, q^{\prime}}\left(l_{2}\right)\|A\|,
$$

since $\frac{1}{r}=\frac{1}{p^{\prime}}-\frac{1}{2}+\frac{1}{q^{\prime}}-\max \left\{\frac{1}{v}, \frac{1}{2}\right\}$. Hence

$$
\left(\sum_{i=1}^{\infty} l_{v}\left(\hat{A} e_{i}\right)^{r}\right)^{1 / r} \leq \pi_{r, p^{\prime}}(I \hat{A}) w_{p^{\prime}}\left(e_{i} ; l_{p}\right) \leq \mu_{2, p^{\prime}}\left(l_{q^{\prime}}\right) \mu_{2, q^{\prime}}\left(l_{2}\right)\|A\| .
$$

\section{REFERENCES}

1. W. Bauhardt, Hilbert-Zahlen von Operatoren in Banachräumen, Math. Nachr. 79 (1977), 181-187.

2. G. Bennett, Inclusion mappings between $l^{p}$-spaces, J. Funct. Anal. 13 (1973), 20-27.

3. __ Schur multipliers, Duke Math. J. 44 (1977), 603-639.

4. B. Carl, Absolut $(p, 1)$-summierende identische Operatoren von $l_{u}$ nach $l_{v}$, Math. Nachr. 63 (1974), 253-360.

5. B. Carl, B. Maurey, and J. Puhl, Grenzordnungen von absolut $(r, p)$-summierenden Operatoren, Math. Nachr. 82 (1978), 205-218.

6. S. Chevet, Sur certain produits tensoriels topologiques d'espaces de Banach, Z. Wahrsch. Verw. Gebiete 11 (1969), 120-138.

7. J. S. Cohen, Absolutely p-summing, p-nuclear operators and their conjugates, Math. Ann. 201 (1973), 177-200.

8. A. Defant and K. Floret, Tensor norms and operator ideals, North-Holland Math. Studies (to appear).

9. A. Grothendieck, Résumé de la théorie métrique des produits tensoriels topologiques, Bol. Soc. Mat. Säo Paulo 8 (1956), 1-79. 
10. G. H. Hardy and J. E. Littlewood, Bilinear forms bounded in space $[p, q]$, Quart. J. Math. Oxford Ser. (2) 5 (1934), 241-254.

11. Her. König, Eigenvalue distribution of compact operators, Birkhäuser, Basel and Boston, Mass., 1986.

12. S. Kwapien, Some remarks on $(p, q)$-absolutely summing operators in $l_{p}$-spaces, Studia Math. 29 (1968), 327-337.

13. $\ldots$ A remark on p-absolutely summing operators in $l_{r}$-spaces, Studia Math. 34 (1970), 109-111.

14. J. Lindenstrauss and A. Pelczyński, Absolutely summing operators in $L_{p}$-spaces and their applications, Studia Math. 29 (1968), 275-326.

15. J. Lindenstrauss and L. Tzafriri, Classical Banach spaces, vols. I and II, Springer-Verlag, Berlin, Heidelberg, and New York, 1977/79.

16. B. Maurey, Théorèmes de factorisation pour les opérateurs linéaires à valeurs dans les espaces $L^{p}$, Astérisque, vol. 11, Soc. Math. France, Paris, 1974.

17. __ Application $\Lambda_{p}$-sommantes, Séminaire Maurey-Schwartz, Exposé no. 10. 1974

18. W. Orlicz, Über unbedingte Konvergenz in Funktionenräumen, Studia Math. 4 (1933), 3337 and $41-47$.

19. A. Persson, On some properties of p-nuclear and p-integral operators, Studia Math. 33 (1969), 213-222.

20. G. Pisier, Factorization of linear operators and geometry of Banach spaces, CBMS Regional Conf. Ser. in Math., no. 60, Amer. Math. Soc., Providence, R.I., 1986.

21. A. Pietsch, s-numbers of operators in Banach spaces, Studia Math. 51 (1974), 201-223.

22. __ Operator ideals, North-Holland, Amsterdam, New York, and Oxford, 1980.

23. __ Eigenvalues and s-numbers, Akademische Verlagsgesellschaft, Geest \& Portig, Leipzig, 1987.

24. J. Puhl, Quotienten von Operatorenidealen, Math. Nachr. 79 (1977), 131-144.

25. B. Russo, On the Hausdorff-Young theorem for integral operators, Pacific J. Math. 68 (1977), 241-253.

26. P. Saphar, Produits tensoriels d'espaces de Banach et classes d'applications linéaires, Studia Math. 38 (1970), 71-100.

27. __, Applications $p$ décomposantes et $p$ absolument sommantes, Israel J. Math. 11 (1972), 379-399.

Fachbereich Mathematik, Universität Oldenburg, 2900 Oldenburg, Germany 